Published in final edited form as:

Neurol Clin. 2015 November ; 33(4): 831-846. doi:10.1016/j.ncl.2015.07.004.

\title{
Spinal Muscular Atrophy
}

\author{
Stephen J. Kolb, M.D., Ph.D. [Assistant Professor] and \\ Department of Neurology and Department of Biological Chemistry and Pharmacology, The Ohio \\ State University Wexner Medical Center, Columbus, $\mathrm{OH}$
}

John T. Kissel, M.D. [Professor and Chairman]

Department of Neurology, The Ohio State University Wexner Medical Center, Columbus, $\mathrm{OH}$

Stephen J. Kolb: stephen.kolb@osumc.edu

\begin{abstract}
Incidence-The incidence of SMA is 1:11,000 live births [1].

Prevalence-The prevalence of the carrier state is approximately 1 in 54 [1].

Severity-The clinical severity of SMA correlates inversely with SMN2 gene copy number and varies from an extreme weakness and paraplegia of infancy to a mild proximal weakness of adulthood.

Natural History-The natural history of SMA is complex and variable. For this reason, clinical subgroups have been defined based upon best motor function attainment during development. Type 1 SMA infants never sit independently. Type 2 SMA children sit at some point during their childhood, but never walk independently. And Type 3 SMA children and adults are able to walk independently at some point in their childhood.
\end{abstract}

\section{Keywords}

Spinal Muscular Atrophy; Motor Neuron; Survival Motor Neuron Gene; SMN1; SMN2

\section{Introduction}

The term spinal muscular atrophy (SMA) refers to a group of genetic disorders all characterized by degeneration of anterior horn cells and resultant muscle atrophy and weakness. The most common SMA, accounting for over $95 \%$ of cases, is an autosomal recessive disorder that results from a homozygous deletion or mutation in the $5 \mathrm{q} 13$ survival

CORRESPONDING AUTHOR. AUTHOR CONTACT INFORMATION. Stephen. J. Kolb, M.D., Ph.D., Assistant Professor,, Departments of Neurology and Molecular \& Cellular Biochemistry, Wexner Medical Center at The Ohio State University, Hamilton Hall, Room 337B, 1645 Neil Avenue, Columbus, OH 43210-1228.

Publisher's Disclaimer: This is a PDF file of an unedited manuscript that has been accepted for publication. As a service to our customers we are providing this early version of the manuscript. The manuscript will undergo copyediting, typesetting, and review of the resulting proof before it is published in its final citable form. Please note that during the production process errors may be discovered which could affect the content, and all legal disclaimers that apply to the journal pertain.

DISCLOSURE STATEMENT

Disclose any relationship with a commercial company that has a direct financial interest in subject matter or materials discussed in article or with a company making a competing product. If nothing to disclose, please state "The Authors have nothing to disclose." SJK has received compensation for consulting from Biogen, F. Hoffman-La Roche, Jeffries LLC, and the Deerfield Institute. 
of motor neuron (SMN1) gene. In a large, multi-ethnic study to test the feasibility of highthroughput genetic testing for SMA carriers, the overall carrier frequency was one in 54 with an incidence of 1 in 11,000 [1]. The severity of SMA is highly variable and the clinical features can be classified into four main phenotypes on the basis of age of onset and maximum motor function achieved [2]. There is no cure for SMA, however an understanding of the molecular genetics of SMA has led to the development of pre-clinical models and numerous potential therapeutic approaches [3-5]. There is great excitement in the SMA field as these therapeutic approaches have recently entered early phase clinical trials.

Paired with the excitement of an active therapeutic pipeline in SMA has been a focus upon understanding the natural history of this disorder as well as early diagnosis and clinical intervention. This has led to the development of clinical standards of care [6, 7]. The natural history of the most severe form of spinal muscular atrophy (type 1) has been the subject of particular attention and is characterized by a rapid loss of motor and respiratory function in the first year of life [8]. Studies have shown that survival beyond one year in these infants can be improved to $70 \%$ or more with proactive use of non-invasive ventilatory support and enteral feeding [9-11]. In contrast, studies on the natural history of the milder forms of SMA (type 2 and type 3 ) have shown little reduction in motor and respiratory function during the course of a single year $[12,13]$.

This chapter will focus upon the clinical manifestations of SMA and how it relates to the molecular genetics and pathogenesis of the disease. We will discuss genetic testing and will review the clinical management of SMA with particular attention to aspects of care and methods of assessment that can be utilized in clinical practice and as clinical trial outcome measures. We will also review current therapeutic approaches and highlight current controversies in clinical management, newborn screening and clinical trial design.

\section{Clinical Features}

The predominant clinical features of SMA are muscle weakness and atrophy. Weakness is usually symmetric with proximal muscles more affected than distal groups as in NP7 (see in this issue "Patterns of Weakness, Classification of Motor Neuron Disease \& Clinical Diagnosis of Sporadic ALS"). Over the last 125 years, reports detailing the clinical manifestations and wide range of clinical severity have all recognized and emphasized the seminal pathology as anterior horn cell degeneration, as well as the pertinent clinical features of symmetrical, proximal predominant extremity weakness that also affects axial, intercostal, and bulbar musculature [14]. The multiple described phenotypes were eventually formalized into a classification scheme at an International Consortium on Spinal Muscular Atrophy sponsored by the Muscular Dystrophy Association (MDA) in 1991 [15]. This classification highlighted three SMA types based on the highest level of motor function (i.e. sitting or standing) and age of onset. Subsequent modifications divided the type 3 category by age of onset, added a type 4 for adult-onset cases, and included a type 0 for patients with prenatal onset and death within weeks $[16,17]$. Although there are degrees of severity even within an individual type, and as many as $25 \%$ of patients elude precise classification, this 
scheme remains relevant in the genetic era and provides useful clinical and prognostic information (Table 1).

\section{SMA type 0}

Spinal muscular atrophy type 0 is used to describe neonates who present with severe weakness and hypotonia with a history of decreased fetal movements. In this case, the weakness is probably of prenatal onset. On examination, infants with type 0 may have areflexia, facial diplegia, atrial septal defects and joint contractures. Respiratory failure is a major concern early on. Life-expectancy is reduced and most are unable to survive beyond 6 months of age (Table 1) $[18,19]$.

\section{SMA type 1}

Infants with type 1 SMA, also known as Werdnig-Hoffman disease, present with hypotonia, poor head control and reduced or absent tendon reflexes prior to 6 months of age. By definition, they never achieve the ability to sit unassisted (Figure 1)(Table 1). The profound hypotonia can manifest as a "frogleg" posture when lying and poor to absent head control. The weakness in intercostal muscles, with relative sparing of the diaphragm, produces a bell-shaped chest and a pattern of paradoxical breathing sometimes referred to as "bellybreathing". Infants with type 1 SMA develop tongue and swallowing weakness and tongue fasciculations are often present. Facial weakness does develop, although this is usually not manifest early in the course of the disease. As the tongue and pharyngeal muscles weaken, these infants are at risk of aspiration and failure to thrive. Infants with type 1 SMA usually develop respiratory failure prior to 2 years of life [8,20,21]. Despite the profound weakness, cognition in these infants is normal in infants with type 1 SMA who are often alert, attentive and bright at the time of diagnosis.

\section{SMA type 2}

Children with type 2 SMA are able to sit unassisted at some point during their development, however they are never able to walk independently (Figure 2)(Table 1). This intermediate form of SMA tends to manifest as progressive proximal leg weakness that is greater than weakness in the arms. There is hypotonia and areflexia on examination. Many of the comorbidities in this patient population are related to the orthopedic complications of bone and joint development in the setting of muscular weakness and progressive scoliosis, joint contractures and ankylosis of the mandible may develop. The combination of scoliosis and intercostal muscle weakness can also result in significant restrictive lung disease. In these children, cognition is normal [22].

\section{SMA type 3}

Children and adults with type 3 SMA, also referred to as Kugelberg-Welander disease, are able to walk unassisted at some point during their lifetime (Figure 3)(Table 1). They present with progressive proximal weakness of the legs more than the arms. The leg weakness may necessitate the need of a wheelchair at some point. Unlike, type 2, these individuals are mostly spared the comorbities of scoliosis and have little or no respiratory muscle weakness. Cognition and life expectancy are not altered in this group [17, 23]. 


\section{SMA type 4}

At the mild end of the continuum are individuals classified as having SMA type 4 . They represent $<5 \%$ of SMA cases and have the mildest form of the disease. These individuals are ambulatory and they are similar to type 3 , however onset is in adulthood, often considered to present at age 30 or later but can be of juvenile onset. [21, 24].

Case vignette-This 37 y.o. police officer presented with a chief complaint of leg weakness. He dated his symptoms back 12 months when he noticed difficulty going up stairs and getting out of deep chairs. To more detailed questioning, he recalled that 10 years previously while in the police academy he had had to push off his knees with his arms to stand up after shooting practice from a kneeling position. He complained of muscle cramps and occasional "twitches" in his legs, but denied arm or bulbar weakness or sensory problems. He had been evaluated at an outside hospital where an EMG revealed "diffuse denervation" a diagnosis of ALS was made, he was told to "get his affairs in order".

Examination revealed intact cranial nerves, symmetric 4+ weakness in the shoulder abductors, symmetric 2 grade weakness in the hip flexors and extensors and 4- knee flexors and extensor strength. Distal groups were grade 5 in both the arms and legs and sensation and reflexes were normal. Repeat EMG confirmed diffuse denervation and SMN genetic testing revealed a homozygous deletion in SMN1 gene with 5 copies of SMN2.

Comment-In this patient with SMA type 4, the symmetric weakness, preferential involvement of hip flexors, and lack of upper motor neuron signs all suggested that ALS was not the correct diagnosis. The 5 copies of SMN2 account for the mild phenotype. Eight years after diagnosis, the patient is still working as a police officer doing desk duties.

\section{Molecular Genetics}

Prior to the discovery of the genetic etiology, SMA presented a riddle in regards to severity: how can one gene defect result in such a wide range of clinical severity? The solution to this riddle began with the discovery by the Melki laboratory in 1995 that $95 \%$ of cases of SMA, irrespective of type, are caused by a homozygous deletion in the SMN1 gene on chromosome 5q13 [25]. In humans, two forms of the $S M N$ gene exist on each allele: a telomeric form (SMN1) and a centromeric form (SMN2) (Figure 4). Transcription of the $S M N 1$ gene produces full-length mRNA transcripts that encode the SMN protein. The SMN2 gene is identical to the $S M N 1$ gene with the exception of a C to T substitution in an exonic splicing enhancer that results in the exclusion of exon 7 during transcription. The resultant, truncated protein is not functional and is rapidly degraded. Critically, the exclusion of exon 7 from SMN2 mRNAs is not complete, and so a small fraction of the total mRNA transcripts $(\sim 10-15 \%)$ arising from the $S M N 2$ gene do contain exon 7, which encodes the normal SMN protein (Figure 4).

All patients with SMA lack a functioning SMN1 gene and are thus dependent upon their $S M N 2$ gene, however inefficient, to produce the SMN protein necessary for survival. Thus, SMA is caused by a deficiency of the SMN protein that, for reasons still unknown, results in selective motor neuron loss. The riddle of severity was solved by the variability of $S M N 2$ 
gene copy number that was found in SMA patients[26]. Several subsequent genotype/ phenotype analyses confirmed a positive correlation between $S M N 2$ copy number and milder phenotype [27]. Although SMN2 copy number is now known to be the primary determinant of SMA severity, it is clearly not the only phenotypic modifier. Prior and colleagues described three adult patients with mild $3 \mathrm{~b}$ phenotypes and only 2 copies $S M N 2$, a seemingly incongruous finding that was explained by the fact that these individuals had a c.859G $>C$ exon 7 mutation that created an exon splice enhancing element resulting in increased full-length SMN protein production and a milder phenotype [28]. Other modifiers have been described and more are expected as the understanding of the molecular pathogenesis of SMA is refined. Thus, the SMA phenotype cannot always be deduced solely from the $S M N 2$ copy number determination, a fact crucial when performing genetic counseling with patient families. Because clinically validated genetic testing is available, it is feasible to someday add the SMA gene test to newborn screening panels [29, 30]. In order for this to occur, however, clear evidence of an effective therapy for SMA and improved ability to understand genetic modifiers of disease severity is required.

\section{Molecular Pathology}

Although a detailed discussion of the pathogenesis of SMA is beyond the scope of this review, a few comments are in order. SMN is found throughout the cytoplasm and nucleus where it functions as part of a multi-protein complex, the SMN complex, that plays an essential role in spliceosomal small nuclear ribonuclear protein (snRNP) biogenesis and premRNA splicing [31]. snRNP biogenesis is altered in the cells of SMA mice. The SMN protein has also been detected in the axons of motor neurons. These observations have led to a central question: while the SMN protein influences RNA processing functions in all cells, does the protein have an additional, unique function in motor neurons? [32, 33] One parsimonious explanation may be that the downstream consequences of altered RNA processing that result from insufficient expression of SMN are not favorable for motor neuron development, survival or both. In this sense, because the motor neuron transcriptome is unique, a global alteration in splicing, for example, could have a unique effect on the transcriptome of motor neurons. The relevant function(s) of the SMN protein in SMA is an area of active research that holds promise to add to our understanding of basic motor neuron biology which may prove relevant in other disorders affecting the lower motor neuron.

\section{Clinical Management}

Over the past decade, there has been a marked improvement in the ability of clinicians to manage the multiple respiratory, nutritional, orthopedic, rehabilitative, emotional and social problems that develop in the majority of these patients. A notable achievement in this regard was the development of a comprehensive standard of care document by Wang and a collaborating panel of experts that was published in 2007 [6] and is currently being updated. This document established guidelines for managing the multiple expected clinical problems that develop in patients with SMA as they age. 


\section{Pulmonary}

The ultimate cause of death in infants and children with type 1 and 2 SMA is usually respiratory failure. It is critical that a therapeutic relationship with an experience pulmonary specialist familiar with pediatric neuromuscular disorders is established at the time of initial diagnosis. There is early involvement of the expiratory muscles of ventilation with relative sparing of the diaphragm [34, 35]. In infants with type 1 SMA, the early implementation of noninvansive ventilatory support has been show to improve survival and quality of life [10, 36]. Bi-level positive airway pressure, when applied with appropriate pressure settings and mask placement, is well tolerated, does not affect hemodynamic balance and may increase chemosensitivity and improve daytime hypercapnic ventilatory drive [37-39]. Patients with this degree of respiratory weakness also have a weak cough that puts them at increased risk of aspiration and hypoxemia secondary to mucus plugging as well as increased risk of recurrent pulmonary infections. Infants at risk for mucus plugging should be monitored with overnight oximetry during acute illnesses and assisted airway clearance methods, such as manual suctioning, is recommended. Generally, the use of antibiotics should be applied to these infants during any acute illness because fo the risk of pneumonia and associated pulmonary complication $[6,36,40]$.

The goal of pulmonary intervention in type 1 infants is to improve quality of life and not necessarily to prolong life. For example, non-invasive ventilation can prevent and may reverse changes in chest wall compliance and lung development [41]. Ultimately, however, a decision must be made about what to do once non-invasive ventilatory support is not sufficient. The use of a tracheostomy and permanent ventilatory support can be successfully implemented in individuals with SMA [42]. However, a commitment to lifelong, full-time ventilatory support is an individual choice for the child's family which must be discussed in a multi-disciplinary setting, ideally involving a palliative care team [36].

The management of respiratory function in type 2 or non-ambulant SMA children (i.e. type 3 children who with progression of illness eventually lose ambulation) is similar to that of type 1 infants, however the complications are less severe. Physical examination and assessment of cough effectiveness and respiratory muscle function should be routinely monitored. For children who are greater than 5 years of age, forced vital capacity can be routinely monitored and non-invasive ventilatory support can be managed long-term. Nocturnal hypoventilation should also be treated with non-invasive ventilation [38].

\section{Gastrointestinal and nutritional}

Gastrointestinal complications are common in individuals with SMA, and it is not clear if this is due to immobility and nutritional deficiencies or whether there is a primary defect is gastrointestinal mobility [43, 44]. Infants with type 1 SMA often have prolonged feeding times and tire quickly. This reduction in feeding can be the first sign of progressive weakness and can lead to failure to thrive and aspiration. [40]. Gastrointestinal dysfunction includes difficulty feeding and swallowing due to bulbar dysfunction and manifests as tongue weakness, difficulty opening the mouth and poor head control. Other associated problems include gastrointestinal reflux, delayed gastric emptying and constipation [40]. 
These complications are also seen in individuals who, for other reasons, cannot sit or stand, and are less commonly seen in ambulant individuals with SMA.

The management of aspiration associated with feeding and dysphagia includes changing food consistency to include semi-solid and thickened liquids. However, in infants with type 1 SMA, early gastrostomy and laparoscopic Nissen fundoplication (if gastrointestinal reflux is present) is recommended because of the importance of maintaining proper nutrition and to reduce the risk of infection secondary to aspiration [45-47]. The surgery can be performed soon after diagnosis when the infant is healthy so that the infant will not become hungry as oral feeding decreases.

Malnutrition, secondary to decreased oral intake can also be an insidious problem for some type 2 SMA children and adolescents [48]. Malnutrition and periods of fasting should be avoided, since these behaviors may contribute to reduction of muscle mass and subsequent impaired function. In clinical practice, height and weight plots in individuals with SMA can be deceiving due to reduced lean body mass [49]. In fact, high functioning, non-ambulatory individuals with SMA are prone to adiposity, despite their low resting energy expenditure and are at risk of becoming overweight [50,51]. To manage these complications, each child should be evaluated individually during routine visits by a dietitian with the goal of maintain the growth curve and to avoid inadequate or excessive intake. Because of the tendency for decreased bone mineral density with increasing age, adequate intake of vitamin $\mathrm{D}$ and calcium should be provided [52].

\section{Orthopedic and musculoskeletal complications}

Weakness and impaired mobility predispose to numerous musculoskeletal issues. Early recognition and appropriate management are helpful in maintaining function, preventing deterioration in vital capacity and improving quality of life. In non-ambulatory individuals with SMA, contractures are common and regular stretching and bracing programs to preserve flexibility and prevent contractures are the main goals of therapy [53, 54]. Manual and motorized wheelchairs may be initiated as early as 18-24 months of age. Children who are able to bear some weight and have some trunk control may utilize a standing frame or mobile-stander with ankle-foot orthoses. Physical therapy can help to maximize endurance, fitness and safety by incorporating activities such as swimming, aquatic therapy and adaptive sports. Neuromuscular fatigue appears to contribute to functional limitations in individuals with SMA [55]. Wheelchairs and modifications in the environment and at home should be considered to allow for safe accessibility and to optimize independence.

Scoliosis occurs in almost all non-ambulant individuals with SMA [56]. When untreated, scoliosis causes chest-cage deformities with subsequent respiratory restriction. Spinal fusion and bracing are the treatments of choice for scoliosis, however there is no clear consensus for their efficacy [57-60].

\section{Therapeutic Development}

Prior to the 1990s, there were relatively few clinical trials in SMA because there was no clear molecular target. Those studies that were undertaken usually involved pharmacologic 
agents that were repurposed and had shown encouraging results in other diseases characterized by muscle weakness such as amyotrophic lateral sclerosis or muscular dystrophy. Within 5 years of the discovery of the $S M N$ gene, however, animal models of SMA were developed that mimic many of the pathological and electrophysiological changes seen in patients and have formed the cornerstone for all therapeutic developments that followed. Burghes and colleagues found that mice with two copies of human SMN2 on a null smn-/- background are viable with a severe SMA-like phenotype, loss of motor neurons and lifespan of 5 days, whereas mice with eight copies of SMN2 on the same background are normal [61]. This and subsequent murine models, as well as the development of SMA models in zebrafish, Drosophila, and pig have provided "proof of principle" that increasing expression of the full length SMN protein is protective. These models also established superb pre-clinical model systems for screening potential therapies, and permitted in-depth molecular and biochemical studies of disease pathogenesis [62,63].

The elucidation of the genetic and molecular basis of SMA described above, suggested several possible therapeutic approaches based on the general principle of increasing the expression of the SMN protein. These strategies include pharmacologic or gene-based therapies to increase $S M N 2$ expression (leading to more full-length SMN mRNA), antisense oligonucleotide-based therapies to favor incorporation of exon 7 into SMN2-derived mRNA transcripts, and viral mediated therapies to replace the entire $S M N 1$ gene. The development of these approaches is now proceeding at a rapid rate, with human clinical trials using RNAbased oligonucleotide and gene therapy approaches currently underway.

\section{Small Molecule Therapy}

The past decade has witnessed several large SMA drug development projects coordinated by the NINDS, private foundations and the pharmaceutical and biotechnology industries. These projects have focused on developing cell-based, high-throughput assays to screen for candidate small molecules that can increase SMN protein levels. Compounds that increase SMN levels in these assays have been tested in SMA animal models. Through this approach, a diverse set of compounds has been identified that include histone deacetylase (HDAC) inhibitors, aminoglycosides, and quinazolone derivatives. HDAC inhibitors such as valproic acid, sodium butyrate, phenylbutyrate and trichstatin A activate the SMN2 promoter resulting in increased full-length SMN protein. Despite favorable results in mouse models, clinical trials with several of these agents, most notably phenylbutyrate, valproic acid, and hydroxyurea have been disappointing with no substantial clinical benefit demonstrated. Multiple issues related to dosing, duration of therapy, and timing of therapy (i.e. when in the course of the disease therapy is instituted), may have confounded these earlier studies, however there are now many new small molecules that have been discovered and developed specifically for their ability to affect the splicing of the SMN2 gene to increase the amount of full-length SMN mRNA transcript. The development and testing of these compounds in clinical trials is an active area that holds promise for disease modifying activity.

\section{RNA-based Therapy}

Alteration of SMN2 splicing to favor inclusion of exon 7 into the final mRNA transcript and increased expression of the full-length SMN protein is a second promising strategy to treat 
SMA. These approaches target interactions between cis-acting sequence motifs found in the introns and exons of SMN2 pre-mRNA and the various trans-acting splicing factors involved in the regulation of exon 7 splicing. Antisense oligonucleotides (ASOs) are therapeutic RNA molecules designed to bind to their complementary sequences within a targeted intron or exon that can either enhance or disrupt the targeted splicing event. Initial efforts to increase the inclusion of exon 7 from SMN2 pre-mRNA utilized an ASO designed to inhibit a 3' spice site of exon 8 [64]. Since then, additional splice site regulators have been identified and refinements have been made to the chemical stability of ASOs. One such additional splice site regulator is the intron 7 intronic splicing silencer N1 (ISS-N1) in the SMN2 gene [65]. ASO-induced blocking of ISS-N1 strongly enhances exon 7 inclusion in cultured fibroblasts and results in increased SMN1 protein. In vivo efficacy testing of these ASOs resulted in efficient inclusion of exon 7 and increased full-length SMN protein levels in mice [66]. In this first pre-clinical ASO study, systemic administration failed to penetrate the blood brain barrier, however this obstacle was quickly overcome by several groups resulting in increased full-length SMN mRNA and protein in mouse spinal cord when delivered by intracerebroventricular (ICV) injection [67, 68].

There are now a number of variations on the theme of RNA-based therapies for SMA that are in various stages of preclinical development. For example, a Morpholino-based ASO that targets an intronic repressor, Element 1 also improves survival in a mouse model of SMA [69]. Bifunctional RNAs are ASOs that are either covalently linked to a peptide or to a sequence recognized by specific splicing modulators in order to enhance the stability and/or activity of the therapeutic RNA. Lorson and colleagues engineered a bifunctional RNA containing an ASO directed to the intron7/exon 8 junction that also contained a sequence that recruited hnRNP-A1, a splicing factor that prevents exon 8 inclusion, resulting in more exon 7 inclusion [70]. ICV injections of this bifunctional RNA therapeutic molecule and other bifunctional RNAs raised full-length SMN levels in brain [70, 71]. Trans-splicing RNAs (tsRNA) are an additional RNA-based strategy that has been applied to SMA mouse models. Therapeutic tsRNA for SMA is a synthetic RNA molecule that interacts with the endogenous $S M N 2$ pre-mRNA resulting in a hybrid mRNA that has the endogenous mutation spliced out, thus resulting in more full-length SMN mRNA and protein. Preclinical studies with therapeutic tsRNA in SMA mouse models have also been successfully applied [72, 73].

\section{Gene Therapy}

Perhaps the most direct approach to SMA therapy is to correct the fundamental cause of the disorder by replacing the missing SMN1 gene. The successful rescue of a mouse model of severe SMA using a self-complementary adeno-associated viral vector, serotype 9 (scAAV9) by Foust and colleagues was a landmark first step in this direction [74]. In this study, mouse pups were treated on postnatal day 1 with a single intravenous injection of $5 \times$ $10^{11}$ viral scAAV9 particles carrying the SMN1 gene. This resulted in SMN1 expression in $60 \%$ of spinal cord motor neurons and complete rescue of motor function and strength, muscle physiology and life span, so that the treated mice had a normal life span of $>400$ days, compared to a life span of approximately 16 days in the untreated animals. This approach has been reproduced by other groups using both AAV8 and AAV9 constructs to 
deliver SMN1 to motor neurons [75-77]. An important caveat to this approach is that injections done in the mice have their maximal effect on postnatal day 1; the effect falls off rapidly with advancing age so that injections on day 5 had only a partial effect, while injections on postnatal date 10 had no effect. This may mean that therapies designed to increase SMN expression, whether via gene therapy approach or otherwise, in humans will have to be coordinated with early disease detection and immediate institution of therapy, hopefully before clinically significant symptomatology develops. This observation suggests that there may be a critical period in development where sufficient SMN protein is essential for the future health of motor neurons, and further study of this aspect of motor neuron biology may yield great insight into the determinants of motor neuron viability. In a recent report of a porcine model of SMA, investigators were able to demonstrate that gene therapy delivered when piglets were symptomatic was beneficial. [62]

\section{Conclusions}

Spinal muscular atrophy is a motor neuron disease of infancy, childhood and adulthood and the genetics and pathophysiology has received extensive study over the last twenty years. This increased focus has led to an improvement of our understanding of the natural history of the many sub-types of SMA and to the development and distribution of standard-of-care recommendations. The dramatic preclinical results in SMA models systems, has also led to incredible cooperation between clinicians, scientists, government, industry, and volunteer organizations on an international scale to develop the guidelines needed for clinical trial readiness.

The SMA community stands at the threshold of an exciting era of opportunity to translate the unusual success in treating SMA mouse models into effective therapy for SMA patients. Due to the remarkable progress made over the past two decades in understanding the molecular pathogenesis of this disease, investigators are now able to effectively screen potential therapies in vitro, test them in accurate animal models, and then move promising agents forward to clinical trials in patients identified in an early or possibly pre-symptomatic stage of disease. It will be a challenging responsibility to prioritize and advance the most promising therapies forward to clinical trials in an efficient, timely, and safe manner under the guidance of the FDA and other regulatory agencies. Testing any therapy will involve developing outcome measures, biomarkers and an infrastructure to conduct meaningful clinical trials, while simultaneously providing optimal supportive care as these new therapies are being developed.

\section{References}

1. Sugarman EA, et al. Pan-ethnic carrier screening and prenatal diagnosis for spinal muscular atrophy: clinical laboratory analysis of >72,400 specimens. Eur J Hum Genet. 2012; 20(1):27-32. [PubMed: 21811307]

2. Munsat TL, Davies KE. International SMA consortium meeting. (26-28 June 1992, Bonn, Germany). Neuromuscul Disord. 1992; 2(5-6):423-428. [PubMed: 1300191]

3. Kolb SJ, Kissel JT. Spinal muscular atrophy: a timely review. Arch Neurol. 2011; 68(8):979-984. [PubMed: 21482919]

4. Arnold WD, Burghes AH. Spinal muscular atrophy: development and implementation of potential treatments. Ann Neurol. 2013; 74(3):348-362. [PubMed: 23939659] 
5. Lorson CL, Rindt H, Shababi M. Spinal muscular atrophy: mechanisms and therapeutic strategies. Hum Mol Genet. 2010; 19(R1):R111-R118. [PubMed: 20392710]

6. Wang $\mathrm{CH}$, et al. Consensus statement for standard of care in spinal muscular atrophy. J Child Neurol. 2007; 22(8):1027-1049. [PubMed: 17761659]

7. D'Amico A, et al. Spinal muscular atrophy. Orphanet J Rare Dis. 2011; 6:71. [PubMed: 22047105]

8. Finkel RS, et al. Observational study of spinal muscular atrophy type I and implications for clinical trials. Neurology. 2014; 83(9):810-817. [PubMed: 25080519]

9. Finkel RS, et al. Respiratory muscle function in infants with spinal muscular atrophy type I. Pediatr Pulmonol. 2014; 49(12):1234-1242. [PubMed: 24777943]

10. Oskoui M, et al. The changing natural history of spinal muscular atrophy type 1. Neurology. 2007; 69(20):1931-1936. [PubMed: 17998484]

11. Boitano LJ. Equipment options for cough augmentation, ventilation, and noninvasive interfaces in neuromuscular respiratory management. Pediatrics. 2009; 123(Suppl 4):S226-S230. [PubMed: 19420149]

12. Swoboda KJ, et al. Natural history of denervation in SMA: Relation to age, SMN2 copy number, and function. Ann Neurol. 2005; 57(5):704-712. [PubMed: 15852397]

13. Kaufmann P, et al. Observational study of spinal muscular atrophy type 2 and 3 : functional outcomes over 1 year. Arch Neurol. 2011; 68(6):779-786. [PubMed: 21320981]

14. Dubowitz V. Ramblings in the history of spinal muscular atrophy. Neuromuscul Disord. 2009; 19(1):69-73. [PubMed: 18951794]

15. Munsat TL. Workshop Report: International SMA collaboration. Neuromuscul Disord. 1991; 1

16. Russman BS. Spinal muscular atrophy: clinical classification and disease heterogeneity. J Child Neurol. 2007; 22(8):946-951. [PubMed: 17761648]

17. Zerres K, Davies KE. 59th ENMC International Workshop: Spinal Muscular Atrophies: recent progress and revised diagnostic criteria 17-19 April 1998, Soestduinen, The Netherlands. Neuromuscul Disord. 1999; 9(4):272-278. [PubMed: 10399757]

18. Dubowitz V. Very severe spinal muscular atrophy (SMA type 0): an expanding clinical phenotype. Eur J Paediatr Neurol. 1999; 3(2):49-51. [PubMed: 10700538]

19. MacLeod MJ, et al. Prenatal onset spinal muscular atrophy. Eur J Paediatr Neurol. 1999; 3(2):6572. [PubMed: 10700541]

20. Thomas NH, Dubowitz V. The natural history of type I (severe) spinal muscular atrophy. Neuromuscul Disord. 1994; 4(5-6):497-502. [PubMed: 7881295]

21. Zerres K, Rudnik-Schoneborn S. Natural history in proximal spinal muscular atrophy. Clinical analysis of 445 patients and suggestions for a modification of existing classifications. Arch Neurol. 1995; 52(5):518-523. [PubMed: 7733848]

22. von Gontard A, et al. Intelligence and cognitive function in children and adolescents with spinal muscular atrophy. Neuromuscul Disord. 2002; 12(2):130-136. [PubMed: 11738354]

23. Zerres K, et al. A collaborative study on the natural history of childhood and juvenile onset proximal spinal muscular atrophy (type II and III SMA): 569 patients. J Neurol Sci. 1997; 146(1): 67-72. [PubMed: 9077498]

24. Piepers S, et al. A natural history study of late onset spinal muscular atrophy types $3 \mathrm{~b}$ and 4 . J Neurol. 2008; 255(9):1400-1404. [PubMed: 18575920]

25. Lefebvre $S$, et al. Identification and characterization of a spinal muscular atrophy-determining gene. Cell. 1995; 80(1):155-165. [PubMed: 7813012]

26. Lefebvre S, et al. Correlation between severity and SMN protein level in spinal muscular atrophy. Nat Genet. 1997; 16(3):265-269. [PubMed: 9207792]

27. Mailman MD, et al. Molecular analysis of spinal muscular atrophy and modification of the phenotype by SMN2. Genet Med. 2002; 4(1):20-26. [PubMed: 11839954]

28. Prior TW, et al. A positive modifier of spinal muscular atrophy in the SMN2 gene. Am J Hum Genet. 2009; 85(3):408-413. [PubMed: 19716110]

29. Taylor JL, et al. Newborn blood spot screening test using multiplexed real-time PCR to simultaneously screen for spinal muscular atrophy and severe combined immunodeficiency. Clin Chem. 2015; 61(2):412-419. [PubMed: 25502182] 
30. Prior TW, et al. Newborn and carrier screening for spinal muscular atrophy. Am J Med Genet A. 2010; 152A(7):1608-1616. [PubMed: 20578137]

31. Kolb SJ, Battle DJ, Dreyfuss G. Molecular Functions of the SMN Complex. J Child Neurol. 2007; 22(8):990-994. [PubMed: 17761654]

32. Burghes AH, Beattie CE. Spinal muscular atrophy: why do low levels of survival motor neuron protein make motor neurons sick? Nat Rev Neurosci. 2009; 10(8):597-609. [PubMed: 19584893]

33. Pellizzoni L. Chaperoning ribonucleoprotein biogenesis in health and disease. EMBO Rep. 2007; 8(4):340-345. [PubMed: 17401408]

34. Kuru S, et al. An autopsy case of spinal muscular atrophy type III (Kugelberg-Welander disease). Neuropathology. 2009; 29(1):63-67. [PubMed: 18410269]

35. Araki S, et al. Neuropathological analysis in spinal muscular atrophy type II. Acta Neuropathol. 2003; 106(5):441-448. [PubMed: 12898156]

36. Schroth MK. Special considerations in the respiratory management of spinal muscular atrophy. Pediatrics. 2009; 123(Suppl 4):S245-S249. [PubMed: 19420154]

37. Panitch HB. The pathophysiology of respiratory impairment in pediatric neuromuscular diseases. Pediatrics. 2009; 123(Suppl 4):S215-S218. [PubMed: 19420146]

38. Markstrom A, Cohen G, Katz-Salamon M. The effect of long term ventilatory support on hemodynamics in children with spinal muscle atrophy (SMA) type II. Sleep Med. 2010; 11(2): 201-204. [PubMed: 20064745]

39. Nickol AH, et al. Mechanisms of improvement of respiratory failure in patients with restrictive thoracic disease treated with non-invasive ventilation. Thorax. 2005; 60(9):754-760. [PubMed: 15939731]

40. Iannaccone ST. Modern management of spinal muscular atrophy. J Child Neurol. 2007; 22(8):974978. [PubMed: 17761652]

41. Roper H, Quinlivan R, Workshop P. Implementation of "the consensus statement for the standard of care in spinal muscular atrophy" when applied to infants with severe type 1 SMA in the UK. Arch Dis Child. 2010; 95(10):845-849. [PubMed: 19819869]

42. Gilgoff IS, et al. Long-term ventilatory support in spinal muscular atrophy. J Pediatr. 1989; 115(6): 904-909. [PubMed: 2585226]

43. Karasick D, Karasick S, Mapp E. Gastrointestinal radiologic manifestations of proximal spinal muscular atrophy (Kugelberg-Welander syndrome). J Natl Med Assoc. 1982; 74(5):475-478. [PubMed: 7120481]

44. Ionasescu V, Christensen J, Hart M. Intestinal pseudo-obstruction in adult spinal muscular atrophy. Muscle Nerve. 1994; 17(8):946-948. [PubMed: 8041404]

45. Durkin ET, et al. Early laparoscopic fundoplication and gastrostomy in infants with spinal muscular atrophy type I. J Pediatr Surg. 2008; 43(11):2031-2037. [PubMed: 18970936]

46. Yuan N, et al. Laparoscopic Nissen fundoplication during gastrostomy tube placement and noninvasive ventilation may improve survival in type I and severe type II spinal muscular atrophy. J Child Neurol. 2007; 22(6):727-731. [PubMed: 17641258]

47. Birnkrant DJ, et al. Treatment of type I spinal muscular atrophy with noninvasive ventilation and gastrostomy feeding. Pediatr Neurol. 1998; 18(5):407-410. [PubMed: 9650680]

48. Chen YS, et al. Prevalence and risk factors for feeding and swallowing difficulties in spinal muscular atrophy types II and III. J Pediatr. 2012; 160(3):447-451. e1. [PubMed: 21924737]

49. Tilton AH, Miller MD, Khoshoo V. Nutrition and swallowing in pediatric neuromuscular patients. Semin Pediatr Neurol. 1998; 5(2):106-115. [PubMed: 9661244]

50. Sproule DM, et al. Increased fat mass and high incidence of overweight despite low body mass index in patients with spinal muscular atrophy. Neuromuscul Disord. 2009; 19(6):391-396. [PubMed: 19427208]

51. Sproule DM, et al. Adiposity is increased among high-functioning, non-ambulatory patients with spinal muscular atrophy. Neuromuscul Disord. 2010; 20(7):448-452. [PubMed: 20610154]

52. Kinali M, et al. Bone mineral density in a paediatric spinal muscular atrophy population. Neuropediatrics. 2004; 35(6):325-328. [PubMed: 15627939] 
53. Fujak A, et al. Contractures of the lower extremities in spinal muscular atrophy type II. Descriptive clinical study with retrospective data collection. Ortop Traumatol Rehabil. 2011; 13(1):27-36. [PubMed: 21393646]

54. Wang HY, et al. Joint range of motion limitations in children and young adults with spinal muscular atrophy. Arch Phys Med Rehabil. 2004; 85(10):1689-1693. [PubMed: 15468032]

55. Montes J, et al. Six-Minute Walk Test demonstrates motor fatigue in spinal muscular atrophy. Neurology. 2010; 74(10):833-838. [PubMed: 20211907]

56. Haaker G, Fujak A. Proximal spinal muscular atrophy: current orthopedic perspective. Appl Clin Genet. 2013; 6(11):113-120. [PubMed: 24399883]

57. Tangsrud SE, et al. Lung function measurements in young children with spinal muscle atrophy; a cross sectional survey on the effect of position and bracing. Arch Dis Child. 2001; 84(6):521-524. [PubMed: 11369575]

58. Granata $\mathrm{C}$, et al. Spinal muscular atrophy: natural history and orthopaedic treatment of scoliosis. Spine (Phila Pa 1976). 1989; 14(7):760-762. [PubMed: 2772728]

59. Fujak A, et al. Treatment of scoliosis in intermediate spinal muscular atrophy (SMA type II) in childhood. Ortop Traumatol Rehabil. 2005; 7(2):175-179. [PubMed: 17615511]

60. Rodillo E, et al. Scoliosis in spinal muscular atrophy: review of 63 cases. J Child Neurol. 1989; 4(2):118-123. [PubMed: 2785548]

61. Monani UR, et al. The human centromeric survival motor neuron gene (SMN2) rescues embryonic lethality in Smn(-/-) mice and results in a mouse with spinal muscular atrophy. Hum Mol Genet. 2000; 9(3):333-339. [PubMed: 10655541]

62. Duque SI, et al. A large animal model of Spinal Muscular Atrophy and correction of phenotype. Ann Neurol. 2014

63. Schmid A, DiDonato CJ. Animal models of spinal muscular atrophy. J Child Neurol. 2007; 22(8): 1004-1012. [PubMed: 17761656]

64. Lim SR, Hertel KJ. Modulation of survival motor neuron pre-mRNA splicing by inhibition of alternative 3' splice site pairing. J Biol Chem. 2001; 276(48):45476-45483. [PubMed: 11584013]

65. Singh NK, et al. Splicing of a critical exon of human Survival Motor Neuron is regulated by a unique silencer element located in the last intron. Mol Cell Biol. 2006; 26(4):1333-1346. [PubMed: 16449646]

66. Hua Y, et al. Antisense masking of an hnRNP A1/A2 intronic splicing silencer corrects SMN2 splicing in transgenic mice. Am J Hum Genet. 2008; 82(4):834-848. [PubMed: 18371932]

67. Williams JH, et al. Oligonucleotide-mediated survival of motor neuron protein expression in CNS improves phenotype in a mouse model of spinal muscular atrophy. J Neurosci. 2009; 29(24):76337638. [PubMed: 19535574]

68. Hua Y, et al. Antisense correction of SMN2 splicing in the CNS rescues necrosis in a type III SMA mouse model. Genes Dev. 2010; 24(15):1634-1644. [PubMed: 20624852]

69. Osman EY, et al. Morpholino antisense oligonucleotides targeting intronic repressor Element1 improve phenotype in SMA mouse models. Hum Mol Genet. 2014; 23(18):4832-4845. [PubMed: 24781211]

70. Dickson A, Osman E, Lorson CL. A negatively acting bifunctional RNA increases survival motor neuron both in vitro and in vivo. Hum Gene Ther. 2008; 19(11):1307-1315. [PubMed: 19848583]

71. Baughan TD, et al. Delivery of bifunctional RNAs that target an intronic repressor and increase SMN levels in an animal model of spinal muscular atrophy. Hum Mol Genet. 2009; 18(9):1600 1611. [PubMed: 19228773]

72. Coady TH, et al. Development of a single vector system that enhances trans-splicing of SMN2 transcripts. PLoS ONE. 2008; 3(10):e3468. [PubMed: 18941511]

73. Coady TH, Lorson CL. Trans-splicing-mediated improvement in a severe mouse model of spinal muscular atrophy. J Neurosci. 2010; 30(1):126-130. [PubMed: 20053895]

74. Foust KD, et al. Rescue of the spinal muscular atrophy phenotype in a mouse model by early postnatal delivery of SMN. Nat Biotechnol. 2010

75. Passini MA, et al. CNS-targeted gene therapy improves survival and motor function in a mouse model of spinal muscular atrophy. J Clin Invest. 2010; 120(4):1253-1264. [PubMed: 20234094] 
76. Dominguez E, et al. Intravenous scAAV9 delivery of a codon-optimized SMN1 sequence rescues SMA mice. Hum Mol Genet. 2010

77. Valori CF, et al. Systemic delivery of scAAV9 expressing SMN prolongs survival in a model of spinal muscular atrophy. Sci Transl Med. 2010; 2(35):35ra42. 


\section{KEY POINTS}

- $\quad$ Spinal muscular atrophy is the most common genetic cause of infant mortality and is characterized by proximal muscular weakness

- $\quad$ Humans have 2 nearly identical inverted SMN genes (SMN1 and SMN2) on chromosome 5q13 and homozygous deletion of the SMN1 gene results in SMA.

- $\quad$ The SMN2 gene produces mostly a shortened, unstable SMN mRNA and, through alternative splicing, a relatively small amount of full-length, functional SMN mRNA

- $\quad$ The $S M N 2$ gene copy number is a good prognostic biomarker of SMA clinical severity

- $\quad$ Clinical management of SMA is supportive, however current and planned clinical trials designed to increase SMN expression levels in motor neurons hold great promise. 


\section{SYNOPSIS}

Spinal muscular atrophy is an autosomal recessive disorder characterized by degeneration of motor neurons in the spinal cord and caused by mutations in the survival motor neuron 1 gene, SMN1. The clinical severity of SMA is highly variable ranging from a fatal disease of infancy to a disorder causing mild proximal muscle weakness in adults and a normal lifespan. This variability is explained by the presence of the SMN2 gene that produces a fraction of the SMN mRNA transcript produced by the SMN1 gene. An inverse correlation of $S M N 2$ gene copy number and clinical severity exists. Specific treatments are not currently available and clinical management is focused upon multidisciplinary care including pulmonary, gastrointestinal, nutritional and orthopedic supportive care. Great excitement in the treatment of preclinical models of SMA has led to an explosion of SMA clinical trials that hold great promise of effective therapy in the near future. 


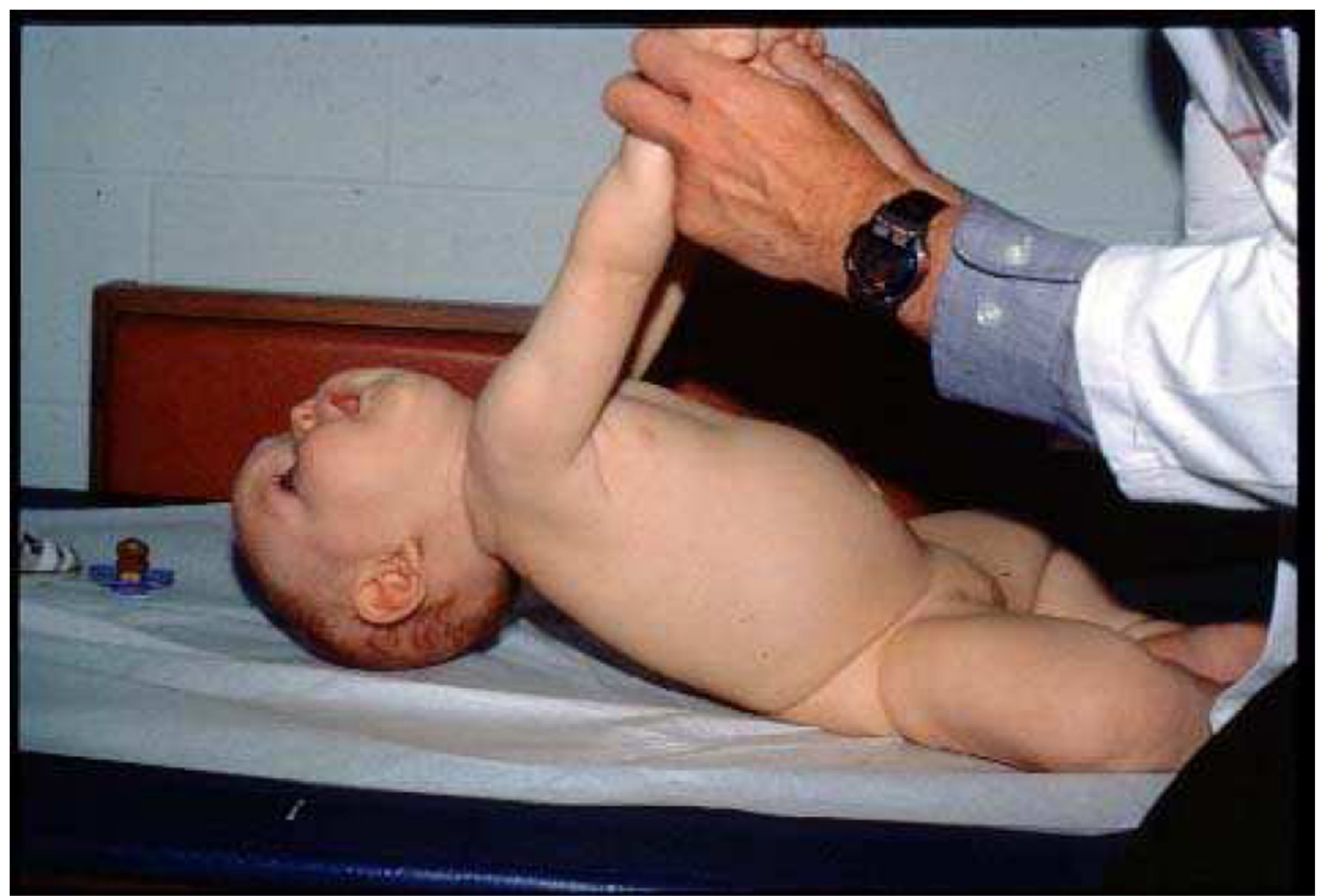

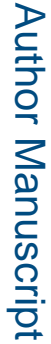

Figure 1.

Spinal muscular atrophy type 1

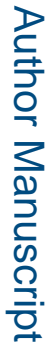




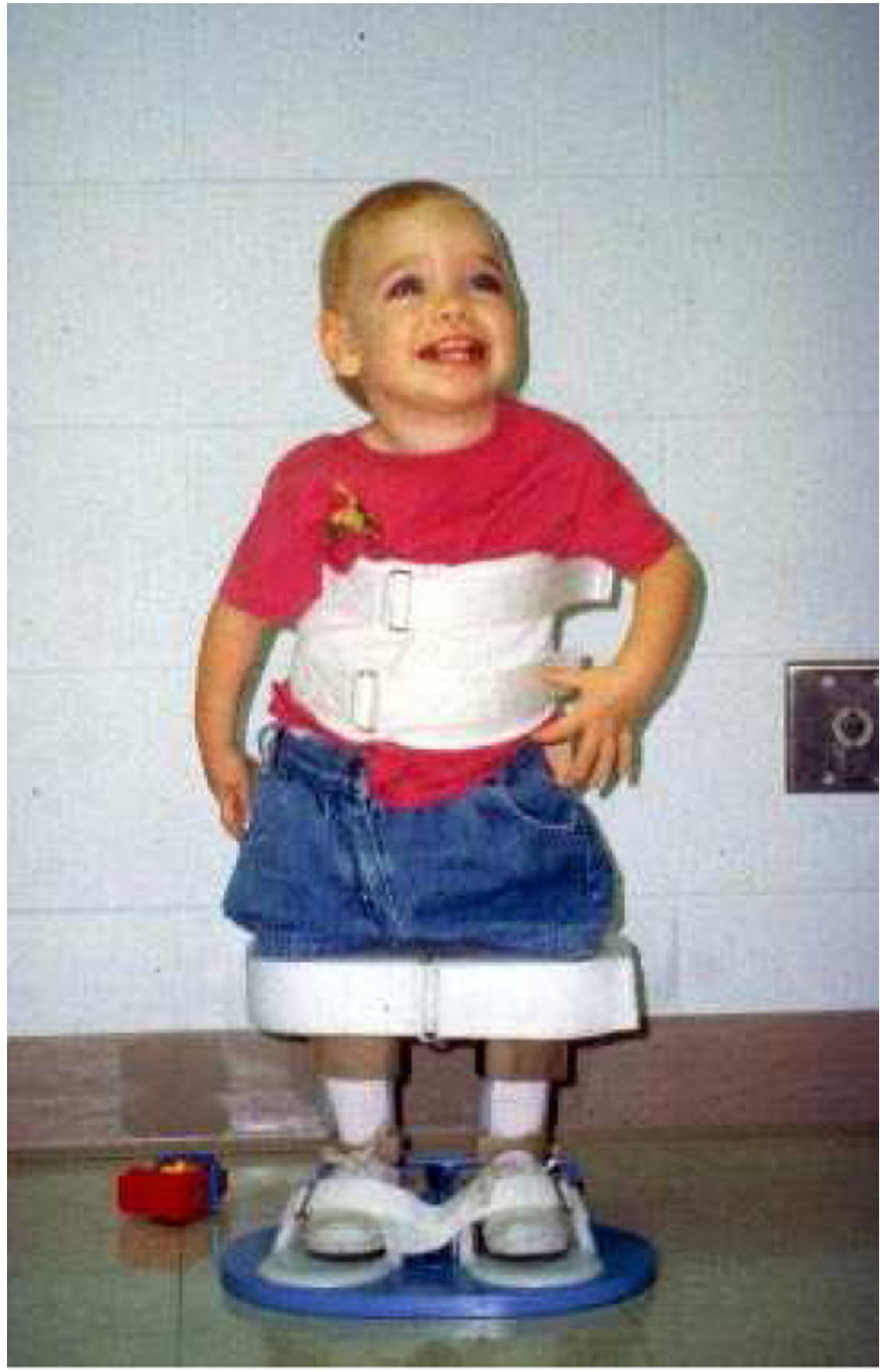

Figure 2.

Spinal Muscular Atrophy type 2 


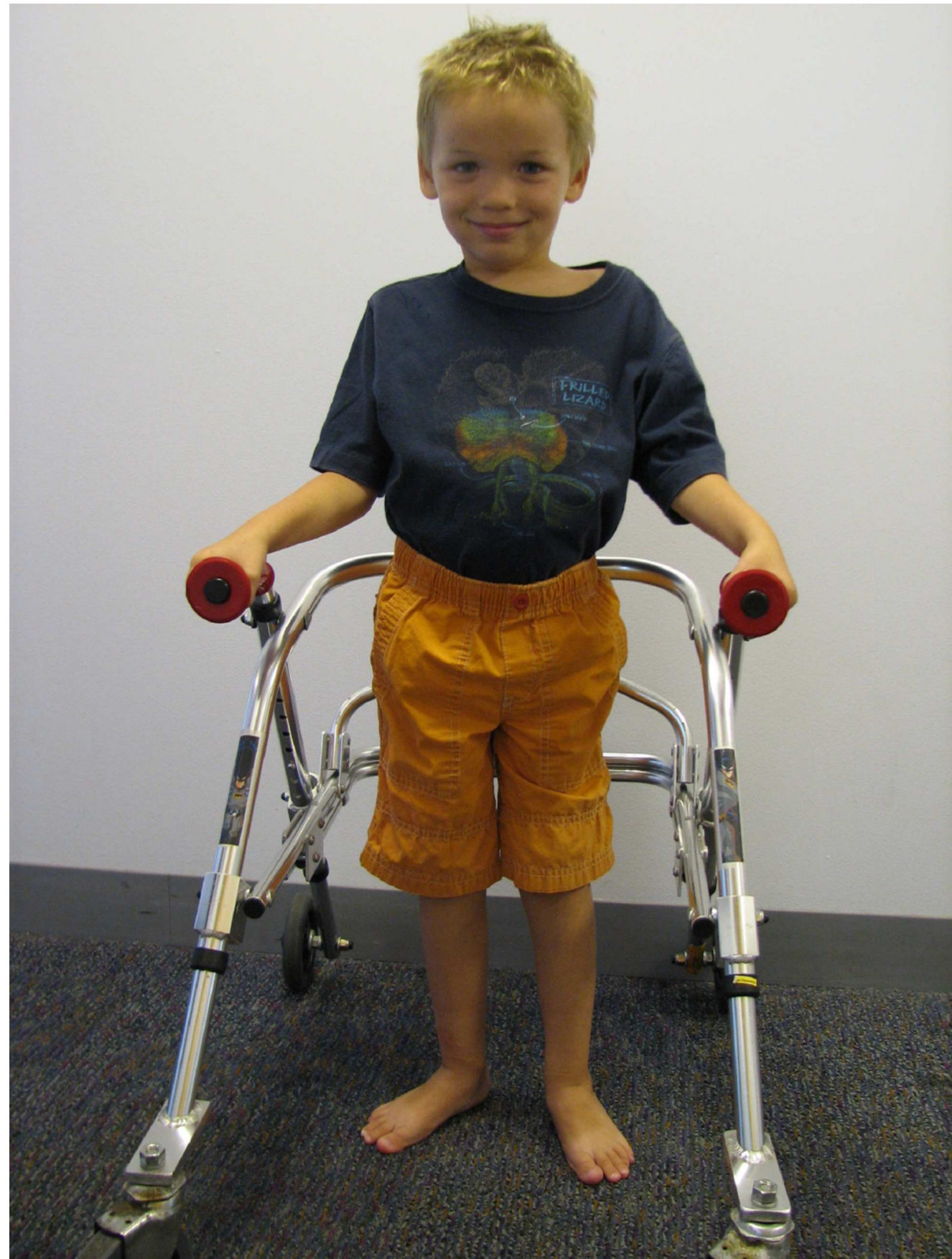

Figure 3.

Spinal Muscular Atrophy type 3 

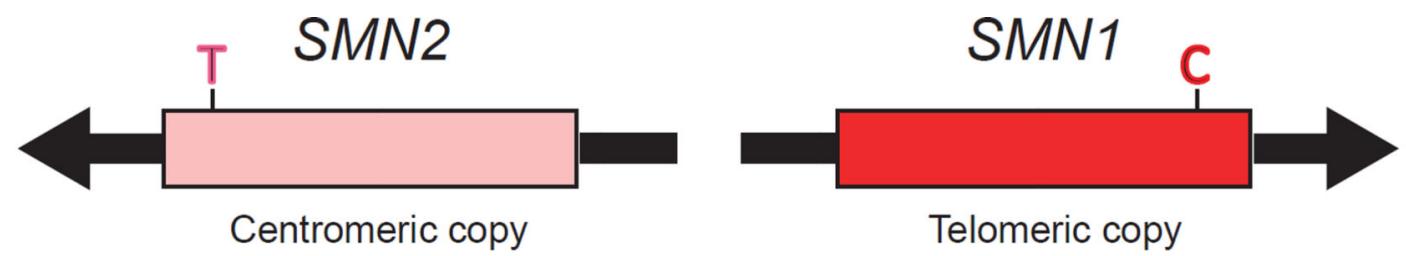

$\sim 15 \%$ exon 7 inclusion


$\sim 85 \%$ exon 7

exclusion
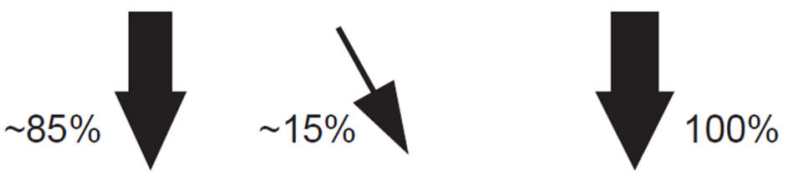

Non-functional


Functional

Figure 4. Schematic of $S M N$ gene

Schematic diagram of the human SMN1 and SMN2 genes and the resultant pre-mRNAs. Patients with SMA have deletions or mutations in both copies of SMN1. The SMN2 gene is expressed, however the majority of the resultant SMN2 pre-mRNA lacks exon 7 because of a C-to-T transition at position 6 of exon 7. The truncated SMN protein is unstable and nonfunctional. A small proportion of full-length mRNA containing exon 7 is produced by from the SMN2 pre-mRNA, however resulting in full-length SMN protein that is functional 
Table 1

\begin{tabular}{|c|c|c|c|c|}
\hline Type & Age of Onset & Highest Function & $\begin{array}{l}\text { Natural Age of } \\
\text { Death }\end{array}$ & SMN2 \# \\
\hline 0 & Prenatal & Resp support & $<1 \mathrm{mo}$ & 1 \\
\hline 1 & $0-6$ mos. & Never sit & $<2 \mathrm{yrs}$ & 2 \\
\hline 2 & $<18$ mos. & Never stand & $>2 \mathrm{yrs}$ & 3,4 \\
\hline 3 & $>18$ mos. & Stand alone & Adult & \\
\hline $3 a$ & 18 mos. - 3 years & Stand alone & Adult & 3,4 \\
\hline $3 b$ & $>3$ years & Stand alone & Adult & 4 \\
\hline 4 & $>21$ years & Stand alone & Adult & $4-8$ \\
\hline
\end{tabular}

